University of Zurich

Department of Economics

Working Paper Series

ISSN 1664-7041 (print)

ISSN 1664-705X (online)

Working Paper No. 207

\title{
An Experimental Test of the Anscombe-Aumann Monotonicity Axiom
}

Florian Schneider and Martin Schonger

Revised version, May 2017 


\title{
AN EXPERIMENTAL TEST OF THE ANSCOMBE-AUMANN MONOTONICITY AXIOM
}

\author{
FLORIAN H. SCHNEIDER AND MARTIN SCHONGER
}

\begin{abstract}
Most models of ambiguity aversion satisfy Anscombe-Aumann's Monotonicity axiom. Monotonicity imposes separability of preferences across events that occur with unknown probability. We construct a test of Monotonicity by modifying the Allais paradox to a setting with both subjective and objective uncertainty. Two experimental studies are conducted: while study 1 uses U.S. online workers and a natural source of ambiguity, study 2 employs European students and an Ellsberg urn. In both studies, modal behavior violates Monotonicity in a specific, intuitive way. Overall, our data suggest that violations of Monotonicity are as prevalent as violations of von Neumann-Morgenstern's Independence axiom.
\end{abstract}

\section{INTRODUCTION}

Since Ellsberg (1961) pioneered the concept of ambiguity aversion, both theorists and experimenters have taken a keen interest in the concept. Ambiguity aversion is usually studied in the Anscombe-Aumann (1963) framework. Anscombe-Aumann proposed a monotonicity axiom. Their Monotonicity axiom prescribes that if two acts differ only on a single state, then the preference between these two acts is given by the preference between the lotteries that are assigned to that state. While both this description of the axiom and its name suggest that it is a mere dominance axiom, Monotonicity is a separability axiom like von Neumann-Morgenstern's Independence axiom. Although Monotonicity is assumed in the majority of models of ambiguity aversion and is controversial, it has not been tested so far. This paper provides an experimental approach to test Monotonicity and then employs it.

Apart from basic choice theoretic axioms like transitivity and continuity, Monotonicity seems to be the most common axiom employed in models of ambiguity aversion. Within the Anscombe-Aumann

Schneider: Department of Economics, University of Zurich, florian.schneider2@econ.uzh.ch. Schonger: Center for Law and Economics, ETH Zurich, mschonger@ethz.ch.

For helpful discussions we would like to thank Björn Bartling, Michael H. Birnbaum, Marie-Charlotte Gütlein, Andreas Haller, Glenn Harrison, Damian Kozbur, Felix Kübler, Jörg Oechssler, Ivo Schurtenberger, Stefan Trautmann, Peter Wakker, Roberto A. Weber, and seminar participants at the European Economic Association, Nordic Conference on Behavioral and Experimental Economics, Conférence Universitaire de Suisse Occidentale, Foundations of Utility and Risk, Royal Economic Society, Berlin Behavioral Economics Seminar, Schweizerische Gesellschaft für Volkswirtschaft und Politik, Toulouse School of Economics, Universities of Tübingen and Heidelberg, and ETH Zurich. Gabriel Gertsch, Sean Hofland and Himanshu Jain provided excellent research assistance. Financial support from the Chair of Gérard Hertig is gratefully acknowledged.

A previous version of this paper was titled "Allais at the Horse Race: Testing Models of Ambiguity Aversion." 
framework, models of ambiguity aversion that satisfy Monotonicity include Multiple priors (Gilboa and Schmeidler, 1989), the Rank-Dependent/Choquet Model (Schmeidler, 1989), Smooth ambiguity preferences (Klibanoff, Marinacci, and Mukerji, 2005), Variational preferences (Maccheroni, Marinacci, and Rustichini, 2006), Confidence-function preferences (Chateauneuf and Faro, 2009), Vector Expected Utility (Siniscalchi, 2009), Uncertainty-averse preferences (Cerreia-Vioglio, Maccheroni, Marinacci, and Montrucchio, 2011), MBA-preferences (Cerreia-Vioglio, Ghirardato, Maccheroni, Marinacci, and Siniscalchi, 2011), monotone Mean-dispersion preferences (Grant and Polak, 2013), and Hedging preferences (Dean and Ortoleva, forthcoming). There are far fewer models that do not impose Monotonicity; they include non-monotone Mean-dispersion preferences (Grant and Polak, 2013), and Bommier's (2016) dual approach.

Monotonicity is a controversial axiom: Some see it as one of the "basic tenets of rationality under ambiguity" (Cerreia-Vioglio, Ghirardato, Maccheroni, Marinacci, and Siniscalchi, 2011), or "a basic rationality axiom" (Gilboa and Marinacci, 2013). Skiadas (2013) assumes Monotonicity, but calls it "not an innocuous assumption". Others view Monotonicity as untenable for non-expected utility decision-makers: Non-expected utility implies that preferences are not separable across events that occur with known probability, yet Monotonicity implies that preferences are separable across events that occur with unknown probability. Wakker (2010 p.301f.; 2011) argues that under non-expected utility, separability across ambiguous events is "undesirable", "implausible", and "unreasonable". Machina (2009) takes the view that "the phenomenon of ambiguity aversion is intrinsically one of nonseparable preferences across mutually exclusive events."

This paper proposes a thought experiment that directly tests Monotonicity. The thought experiment is an adaptation of the classic Allais paradox. Recall that the Allais paradox is set in a world of purely objective uncertainty, and tests expected utility, specifically the von Neumann-Morgenstern Independence axiom. Previous authors (MacCrimmon and Larsson, 1979; Tversky and Kahneman, 1992; Wu and Gonzalez, 1999) have adapted the Allais paradox to a setting of purely subjective uncertainty, where it becomes a test of Subjective Expected Utility. We adapt the Allais paradox to a setting of both objective and subjective uncertainty. The so-modified Allais paradox tests Monotonicity. As sources of objective and subjective uncertainty the thought experiment employs the Anscombe-Aumann metaphors of a horse race and a roulette wheel, respectively. The payoff of the decision-maker depends on the outcome of both sources of uncertainty (see figure 1). The thought experiment, like the Allais paradox, has large hypothetical payoffs. It can be implemented as an incentivized experiment by scaling down the payoffs, and employing practical sources of uncertainty such as Ellsberg urns. Building a test of the descriptive validity of Monotonicity on the 
Allais paradox is advantageous: The extensive literature on the Allais paradox can inform the scaling of the payoffs, and can serve to benchmark the rate of violations. The Allais literature also suggests potential causes of violations, e.g. the certainty effect. While the main purpose of the thought experiment is to suggest incentivized tests, it is also useful in its hypothetical form: Some have understood the Allais paradox as a critique of the view that Independence is a prerequisite of rationality (e.g. Slovic and Tversky, 1974; Allais, 1979 chapter II 3.4; Loomes and Sugden, 1982; Machina, 1989). Similarly, our thought experiment facilitates discussions of whether Monotonicity is a prerequisite of rationality.

There is an extensive experimental literature on ambiguity attitudes, for recent reviews see Hey (2014) and Trautmann and van de Kuilen (2015). However, we know of no experiments testing the Monotonicity axiom. As for thought experiments, most closely related to ours are a few recent thought experiments on separability across ambiguous events: Five thought experiments by Machina (2014) demonstrate that separability restricts variation in ambiguity attitudes at low versus high outcomes. Wakker (2010, p.302) employs a thought experiment in order to argue that separability is implausible for ambiguity averse decision-makers. Bommier (2016) suggests another thought experiment making this point. All these thought experiments are critiques of separability, but none of them directly tests Monotonicity. As a practical matter, these thought experiments are difficult to implement since they require exact measurement of certainty equivalents or a method for implementing payoffs in utils.

We conduct two incentivized studies implementing our thought experiment. Study 1 uses a subject pool of online workers, a real-world lottery as the source of objective uncertainty, and weather in a foreign city for subjective uncertainty. Study 2 takes place in the laboratory with student subjects and employs physical urns. Both studies find that about half of all participants violate Monotonicity, and overwhelmingly do so in a specific, intuitive way. The hypothesis that these violations are due to random errors is easily and robustly rejected in both studies. The specific pattern of violations we find mirrors the pattern that is commonly found in Allais paradox experiments. To be able to assess and interpret the frequency of violations of Monotonicity, it is useful to compare it to the frequency of violations of Independence in the same subject pools. Hence both studies also confront subjects with the original Allais paradox. Violations of Independence are about as common as violations of Monotonicity in both studies. In addition, violations of Independence and Monotonicity are positively correlated. Study 2 also includes a consistency check by repeatedly posing the Monotonicity test. We find that among consistent participants violations of Monotonicity are more prevalent than in the full sample. Overall, we conclude that violations 
1A : \$1 Million with certainty.

1B : $\begin{cases}\$ 0 & \text { Horse 1-11 wins and roulette stops on } 1 . \\ \$ 1 \text { Million } & \text { Horse 12-100 wins. } \\ \$ 5 \text { Million } & \text { Horse 1-11 wins and roulette stops on 2-11. }\end{cases}$

$2 \mathrm{~A}: \begin{cases}\$ 0 & \text { Horse 12-100 wins. } \\ \$ 1 \text { Million } & \text { Horse 1-11 wins. }\end{cases}$

2B : $\begin{cases}\$ 0 & \text { Horse 1-11 wins and roulette stops on 1, } \\ \$ 5 \text { Million } & \text { or Horse 12-100 wins. }\end{cases}$

Figure 1. Thought experiment

of Monotonicity are common and genuine. Knowledge of this phenomenon is important when developing and evaluating descriptive models of ambiguity non-neutrality, as our findings suggest that universally assuming Monotonicity is problematic.

The next section introduces our thought experiment. Section 3 reviews the Monotonicity axiom. Section 4 describes the experimental studies. Section 5 concludes.

\section{A thought Experiment}

Our thought experiment uses the classic Anscombe-Aumann story of a horse race and a roulette wheel. 100 horses numbered from 1 to 100 are starting. The decision-maker does not know the probability that a particular horse will win. At the same time as the horses are running, the roulette wheel is spun. The roulette wheel has 11 equiprobable fields numbered from 1 to 11 . As shown in figure 1, the decision-maker is confronted with two choice situations, 1 and 2 . In each choice situation she has the choice between two options, A and B. In choice situation 1 an intuitively plausible choice, in line with the certainty effect, might be to choose 1A, which is a million for sure, over $1 \mathrm{~B}$, where there is a chance of not winning anything. By contrast in choice situation 2 both bets feature a danger of winning nothing, thus the chance of winning $\$ 5$ Million in bet $2 \mathrm{~B}$ may make that bet more attractive than bet $2 \mathrm{~A}$. Observation 1 in the next section clarifies that this intuitive choice pattern violates Monotonicity. From now on we refer to this choice pattern as the intuitive paradoxical choice pattern.

Compare the thought experiment to the Allais paradox (Allais, 1953), reproduced in figure 2. Recall that in the Allais paradox all uncertainty is objective. Thus in the metaphors of the AnscombeAumann framework, the Allais paradox only features a roulette wheel (albeit with more fields), but 


$$
\begin{aligned}
& \text { IA : } \$ 1 \text { Million with certainty. } \\
& \text { IB : } \begin{cases}\$ 0 & \text { Roulette stops on } 1 . \\
\$ 1 \text { Million } & \text { Roulette stops on } 2-90 . \\
\$ 5 \text { Million } & \text { Roulette stops on } 91-100 .\end{cases} \\
& \text { IIA : } \begin{cases}\$ 0 & \text { Roulette stops on 1-89. } \\
\$ 1 \text { Million } & \text { Roulette stops on } 90-100 .\end{cases} \\
& \text { IIB : } \begin{cases}\$ 0 & \text { Roulette stops on 1-90. } \\
\$ 5 \text { Million } & \text { Roulette stops on 91-100. }\end{cases}
\end{aligned}
$$

FIGURE 2. Allais paradox

no horse race. Our thought experiment differs from the Allais paradox only in that it assigns some uncertainty to a subjective source. Therefore, a decision-maker who does not distinguish between objective and subjective uncertainty, i.e. a probabilistically sophisticated one, views our thought experiment as an Allais paradox. If the decision-maker moreover assigns equal probabilities to the horses, then she views our thought experiment as the same choice situation as the Allais paradox in figure 2 (for a formal statement see p. 8). If she assigns non-equal probabilities, then the thought experiment corresponds to an Allais paradox with probabilities possibly different from the ones in figure $2 .^{1}$

Again, let us call the pattern of choosing IA over IB and IIB over IIA the intuitive paradoxical choice pattern, as it is analogous to the intuitive paradoxical choice pattern in our thought experiment. The intuitive paradoxical choice pattern is what has often been found in experimental investigations of the Allais paradox ${ }^{2}$, and constitutes a violation of Independence. Monotonicity is discussed in the next section, and we show that it is tested by the modified Allais paradox.

\section{The Anscombe-Aumann Monotonicity Axiom}

To discuss Monotonicity and its implications, we introduce an Anscombe-Aumann framework: There are (monetary) prizes $x$ in an interval $X=[\underline{x}, \bar{x}] \subset \mathbb{R}$. We denote the space of simple probability distributions (lotteries) over $X$ by $\mathcal{L}(X)$, with generic elements $P, Q, R$. Denote the degenerate lottery which puts probability 1 on the prize $x$ by $\delta_{x}$. There is a (finite or infinite) set of states $S$ with generic element $s$, endowed with an algebra $\Sigma$ (events). An act $f$ is a $\Sigma$-measurable

${ }^{1}$ Except if she assigns probabilities such that the event "horse $1-11$ wins" has probability 0 or 1 , then the situations become trivial.

${ }^{2}$ See for example Kahneman and Tversky (1979), Conlisk (1989), and Huck and Müller (2012). 
function $^{3} f: S \rightarrow \mathcal{L}(X)$ such that $f(S)=\{f(s): s \in S\}$ is finite. The set of all acts is denoted by $\mathcal{F}$. Generic acts are $f, g, h$. For all acts $f, g$ in $\mathcal{F}$ and all $\alpha$ in $(0,1)$ let $\alpha f+(1-\alpha) g$ denote the act that delivers the lottery $\alpha f(s)+(1-\alpha) g(s)$ in state $s$. For every act $f$, we denote the coarsest partition of the state space it induces by $\mathcal{E}(f)=\left\{E \in \Sigma \mid E=f^{-1}(P)\right.$ for some $\left.P \in f(S)\right\}$. Note that $\mathcal{E}(f)$ is finite. Given an event $E \in \Sigma$, acts $f$ and $g$, let $f_{E} g$ denote the act which gives $f(s)$ for $s \in E$, and $g(s)$ for $s \in E^{C}$. An act that yields the same lottery $P$ in each state is called constant, and, slightly abusing notation, is denoted by $P$. Throughout we assume that objective and subjective uncertainty are resolved simultaneously. $\succsim$ is a preference order on $\mathcal{F}$ :

Axiom (Weak order). $\succsim$ is complete and transitive.

Anscombe-Aumann (1963) proposed a monotonicity axiom which requires that if the lottery which an act assigns to an event is replaced by a preferred lottery, then the new act must be preferred. The modern literature formulates the Anscombe-Aumann Monotonicity axiom as follows (under Weak order the modern and original formulations are equivalent):

Axiom (Monotonicity). For all acts $f, g$ in $\mathcal{F}$ : if for all states $s$ in $S, f(s) \succsim g(s)$, then $f \succsim g$.

Monotonicity implies that preferences are separable across events. Specifically, Monotonicity implies that for all lotteries $P, Q$, all acts $f, g$, and all events $E$, if $P_{E} f \succ Q_{E} f$ then $P_{E} g \succsim Q_{E} g .{ }^{4}$ If the preference admits a utility representation, Monotonicity essentially ensures that one can find a separable, weakly monotone representation. That is an act can be evaluated by a twostep procedure: There is a preference functional and a real-valued, non-decreasing function. The preference functional could, for example, be expected utility or rank-dependent expected utility (Quiggin, 1982). For each state, the preference functional evaluates the lottery the act yields in that state, independently of what the act yields in other states. For the case of a finite state space, this yields $|S|$ numbers which are then aggregated by the non decreasing function to a single number representing the utility of the act (see Bommier, 2016, also Trautmann and Wakker, 2015).

Monotonicity is distinct from first-order stochastic dominance. A lottery $P$ first-order stochastically dominates a lottery $Q$, if for all $x^{\prime}$ we have $\sum_{x: x \leq x^{\prime}} P(x) \leq \sum_{x: x \leq x^{\prime}} Q(x)$. For the AnscombeAumann setting, Machina and Schmeidler (1995) propose a first-order stochastic dominance axiom:

Axiom (AA-FOSD). For all acts $f, g$ in $\mathcal{F}$ : if for all states $s$ in $S, f(s)$ first-order stochastically dominates $g(s)$, then $f \succsim g$.

\footnotetext{
${ }^{3}$ In this definition, $\mathcal{L}(X)$ is endowed with any $\sigma$-algebra which makes singletons measurable.

${ }^{4}$ A stronger version of separability, " $P_{E} f \succsim Q_{E} f \Rightarrow P_{E} g \succsim Q_{E} g$ ", implies a slightly stronger version of Monotonicity, which is not satisfied by the Multiple priors model (Gilboa and Schmeidler, 1989).
} 
When restricting the domain of AA-FOSD to constant acts, the standard first-order stochastic dominance axiom (FOSD) results. Monotonicity is structurally similar to AA-FOSD and basically stronger: as long as the decision-maker satisfies FOSD, Monotonicity implies AA-FOSD.

3.1. Monotonicity and our thought experiment. Translating the thought experiment into a formal setting, define the state space to be $S=\{1,2, \ldots, 100\}$, and consider the event $E=\{1, . ., 11\}$. Using a million dollars as the unit, and denoting $P=\left(0 ; \frac{1}{11}, 5 ; \frac{10}{11}\right)$, the acts can be written as $1 \mathrm{~A}=\delta_{1}, 1 \mathrm{~B}=P_{E} \delta_{1}, 2 \mathrm{~A}=\delta_{1 E} \delta_{0}$, and $2 \mathrm{~B}=P_{E} \delta_{0}$. The following observation tells us which preferences are consistent with Monotonicity:

Observation 1. If $\succsim$ satisfies Weak order and Monotonicity, then:

$1 \mathrm{~A} \succsim 1 \mathrm{~B}$ and $2 \mathrm{~A} \succsim 2 \mathrm{~B}$, or, $1 \mathrm{~B} \succsim 1 \mathrm{~A}$ and $2 \mathrm{~B} \succsim 2 \mathrm{~A}$.

The proof is in the appendix. Monotonicity rules out the intuitive paradoxical choice pattern. Hence the thought experiment is a test of Monotonicity.

The famous Ellsberg experiments have two prizes (compare Machina 2014, fn. 3). Our thought experiment has three prizes, like the Allais paradox, Machina's, Wakker's and Bommier's thoughts experiment. Three or more prizes are required to investigate separability. In a two-prize setting, AA-FOSD implies Monotonicity, and FOSD implies Independence: with only two prizes, first-order stochastic dominance induces a complete order on the lotteries. Thus, with only two prizes, it is impossible to test separability without also testing stochastic dominance.

3.2. The Allais paradox and our thought experiment. In this subsection we formally state the observation that a probabilistically sophisticated decision-maker views a test of Independence (the Allais paradox) and a test of Monotonicity (our thought experiment) as the same problem. In order to do so, we need to define probabilistic sophistication. Machina and Schmeidler (1995) provide a definition and an axiomatic justification for probabilistic sophistication in the AA-framework. Adapting their definition, we directly state it as an axiom:

Axiom (Probabilistic sophistication). There exists a probability measure $\mu$ on $(S, \Sigma)$ such that for all acts $f$ in $\mathcal{F}$, with $\mathcal{E}(f)=\left\{E_{1}, \ldots, E_{N}\right\}$ :

$$
f \sim \sum_{i=1}^{N} \mu\left(E_{i}\right) f\left(E_{i}\right) .
$$

Note that $\sum_{i=1}^{N} \mu\left(E_{i}\right) f\left(E_{i}\right)$ is a constant act, constructed by mixing the lotteries $f\left(E_{i}\right)$ with weights corresponding to the subjective probabilities $\mu\left(E_{i}\right)$ of the events $E_{i}$. A probabilistically sophisticated decision-maker acts as if she has a subjective probability distribution over states, and 
she treats objective and subjective probabilities interchangeably. We can now state formally how, given Probabilistic sophistication and informational symmetry, the Allais paradox and our thought experiment are basically the same problem. Probabilistic sophistication means that the decisionmaker has a probability measure $\mu$. Informational symmetry of the horses implies that $\mu(E)=\frac{11}{100}$. By Probabilistic sophistication the decision-maker is indifferent between each act in the thought experiment and the corresponding act in the Allais paradox: $1 \mathrm{~A} \sim \mathrm{IA}, 1 \mathrm{~B} \sim \mathrm{IB}, 2 \mathrm{~A} \sim \mathrm{IIA}$, and $2 \mathrm{~B} \sim \mathrm{IIB}$.

This observation motivates proposition 1 . The purpose of proposition 1 is to illuminate that a probabilistically sophisticated decision-maker views not only these tests as the same problem, but views the axioms these experiments test as similar. Consider the Independence axiom for objective risk, but restrict mixtures to a set $M \subseteq(0,1)$ :

Axiom (Independence over $M$ ). For all constant acts $P, Q, R$, and for all $\alpha$ in $M \subseteq(0,1)$ :

$$
P \succsim Q \Rightarrow \alpha P+(1-\alpha) R \succsim \alpha Q+(1-\alpha) R
$$

Under probabilistic sophistication, Monotonicity is equivalent to Independence over $M_{\mu}$, with $M_{\mu}$ constructed from $\mu$ :

Proposition 1. If $\succsim$ satisfies Weak order and Probabilistic sophistication with probability measure $\mu$, then Monotonicity and Independence over

$M_{\mu}=\{m \in(0,1) \mid m=\mu(E)$ for some $E \in \Sigma\}$ are equivalent.

The proof is in the appendix. Thus (unrestricted) Independence implies Monotonicity. If $M_{\mu}$ is the unit interval, then the converse is true. For $M_{\mu}$ to be the unit interval requires an infinite state space, and a non-atomicity assumption on $\mu$. Note that in the Anscombe-Aumann framework, Independence is usually strengthened to the AA-Independence axiom. Under probabilistic sophistication, however, Independence implies AA-Independence (see lemma 1, p. 23).

3.3. Critiques of separability. In a setting of purely objective uncertainty, separability is captured by the Independence axiom. Independence is often understood as a prerequisite for rationality, but at least since the Allais paradox this has been doubted. Machina (1989) challenges Independence on normative grounds, pointing out that the description of consequences available to the economist might not be deep enough for separability to hold, say, because it leaves out psychological components such as disappointment, and that therefore in such contexts Independence may be normatively inappropriate. Loomes and Sugden (1982) argue that regret and rejoicing are experiences, and thus cannot be rational or irrational. Accordingly, they take the position that if a 
decision-maker experiences such feelings, taking them into account for decision-making should not be seen as irrational. For a detailed critique of this argument see Bleichrodt and Wakker (2015). Our thought experiment shows that such critiques of separability as prerequisite for rationality in a setting of purely objective uncertainty also apply in a setting of mixed subjective/objective uncertainty. In this sense, the thought experiment contributes to discussing Monotonicity from a normative perspective.

Under ambiguity, separability has another, potentially undesirable, implication, namely it rules out some forms of ambiguity aversion: Take two lotteries $P$ and $Q$ with $P \neq Q$ and $P \sim Q$, and a partition of the state space into two non-null events, $E$ and its complement. Consider the act $f=$ $P_{E} Q$. Note that the act $f$ is an ambiguous act, while the constant act $P$ has no ambiguity. Thus one might expect an ambiguity-averse decision-maker to strictly prefer $P$ over $f$. Monotonicity, however, requires the decision-maker to be indifferent. The logic of this example follows Wakker (2010, p. 302). Machina (2014) provides five thought experiments, which demonstrate that separability restricts how ambiguity aversion can differ between high and low outcomes.

\section{EXPERIMENTS}

To find out to what extent the Monotonicity axiom is descriptively valid, and to compare and relate the prevalence of potential violations of Monotonicity to those of Independence, we run two experimental studies. Study 1 is conducted with a subject pool of online workers and employs a natural source of ambiguity, whereas study 2 is run in the lab with undergraduate student subjects and employs physical urns.

\subsection{Study 1 (Online Experiment).}

4.1.1. Procedure. To implement the thought experiment as an experimental test of Monotonicity, we adapt the prizes and the sources of uncertainty as follows: For the non-zero prizes, rather than $\$ 1$ Million and $\$ 5$ Million we use $\$ 4$ and $\$ 5$. Instead of a horse race, the source of subjective uncertainty is weather in a foreign city as in Fox and Tversky (1995). Specifically, for the event $E$ take the event that tomorrow's maximum temperature in Mexico City is "unexpectedly high". We define "unexpectedly high" as $6^{\circ}$ Fahrenheit $\left(3.3^{\circ}\right.$ Celsius) or more above the current forecast. Participants are told the current forecast and are encouraged to check it on an external website, which shows forecasts and past realizations rounded to integer degrees Fahrenheit. For objective uncertainty we use "Texas Pick 3", a real-world U.S. lottery, which produces the numbers between 000 and 999 with

equal probability. In the test of Monotonicity we consider the last digit, in the Allais paradox the last two digits. So we slightly modify the objective probabilities from our thought experiment (10 
instead of 11 equiprobable objective events). With these modifications, denoting $Q=\left(0 ; \frac{1}{10}, 5 ; \frac{9}{10}\right)$, we get the experimental acts $1 \mathrm{~A}=\delta_{4}, 1 \mathrm{~B}=Q_{E} \delta_{4}, 2 \mathrm{~A}=\delta_{4 E} \delta_{0}$, and $2 \mathrm{~B}=Q_{E} \delta_{0}$. Figure 3 shows, using the example of act $1 \mathrm{~B}$, how acts are displayed to participants (the temperature symbols are defined and explained to participants). Note that observation 1 analogously applies to the acts in the experiment. For the Allais paradox, the lotteries in the experiment differ from the original lotteries only in the prizes as described above.

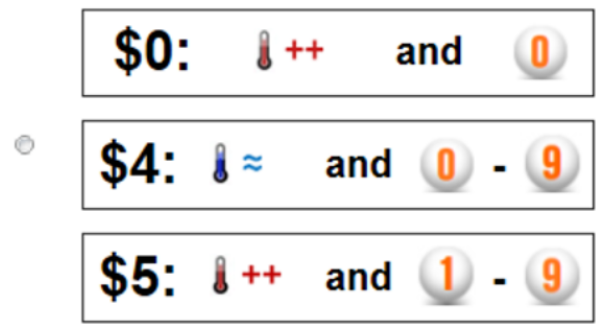

Figure 3. Visual representation of act 1B

Participants were recruited on Amazon Mechanical Turk (mTurk). We restricted participation to U.S. workers. All subjects completed the study within a few hours on the same day. Average earnings were $\$ 0.70$ ( $E^{C}$ realized), average duration was 10 minutes, implying an hourly wage of $\$ 4.20$.

The sequence of the experiment is as follows: the format of the acts is explained, including links to the external weather forecast website and the Texas Pick 3 website, followed by three understanding questions. As a further test of participants' understanding, the subsequent screen offers a choice between two acts, where one act dominates the other in the AA-FOSD sense. On this screen, as well as the Monotonicity and Allais paradox screens, subjects are given a choice between two acts, which are presented in random order. The next two screens each present a choice situation of the Monotonicity test, where the order of the screens (choice situations) is random. This is followed by the two Allais paradox choice situations, which are again displayed in random order. We also measured participants' beliefs about the probability of the events. We use the Random Lottery Incentive (RLI) system to make hedging impossible. A demographic survey concludes the study. ${ }^{5}$

4.1.2. Results. Data from $N=552$ participants was collected. Table 1i gives participants' choices in the Monotonicity test. Consider the first row of results, which concerns the full sample. Choices in columns $\mathrm{AA}$ and $\mathrm{BB}$ do not violate Monotonicity, choices in columns $\mathrm{AB}$ and $\mathrm{BA}$ do. The modal choice of participants violates Monotonicity in a particular way: 38.8 percent of participants exhibit the intuitive paradoxical choice pattern AB. Violations of Monotonicity are not random,

\footnotetext{
${ }^{5}$ The web appendix supplies screenshots and a detailed description of the experiment.
} 
rather, there is an asymmetric pattern, as violations in the opposite way are much rarer with 9.2 percent. Following Conlisk (1989), we test whether violations are the result of symmetric, random participant error. We can reject this hypothesis at all conventional significance levels $\left(\mathrm{Z}=11.06, \mathrm{p}\right.$-value $\left.<10^{-28}\right)$. The following rows repeat the analysis for different subsamples as robustness checks. The second row considers only participants who do not violate AA-FOSD. The next four rows split the sample by duration. The picture which emerges is that the distribution of violations is very similar across subsamples. Table 5i (appendix, p.25) finds robustness across further subsamples.

TABLE 1. Violations of Monotonicity and Independence (in percent)

\begin{tabular}{|c|c|c|c|c|c|c|}
\hline & \multicolumn{2}{|c|}{ SATISFY } & \multicolumn{2}{|c|}{ ViOLATE } & \multirow[b]{2}{*}{ Conl.-Z } & \multirow[b]{2}{*}{$\mathrm{N}$} \\
\hline & $\mathrm{AA}$ & $\mathrm{BB}$ & $\mathrm{AB}$ & $\mathrm{BA}$ & & \\
\hline \multicolumn{7}{|l|}{ (i) Monotonicity } \\
\hline Full sample & 36.8 & 15.2 & 38.8 & 9.2 & 11.06 & 552 \\
\hline Satisfy AA-FOSD & 35.2 & 17.0 & 38.7 & 9.1 & 9.41 & 395 \\
\hline Duration 1. quartile & 37.0 & 14.5 & 39.9 & 8.7 & 5.85 & 138 \\
\hline Duration 2. quartile & 36.2 & 14.5 & 38.4 & 10.9 & 4.99 & 138 \\
\hline Duration 3. quartile & 36.2 & 14.5 & 39.1 & 10.1 & 5.31 & 138 \\
\hline Duration 4. quartile & 37.7 & 17.4 & 37.7 & 7.2 & 5.97 & 138 \\
\hline \multicolumn{7}{|l|}{ (ii) Independence } \\
\hline Full sample & 23.0 & 26.4 & 35.3 & 15.2 & 6.92 & 552 \\
\hline
\end{tabular}

A similar pattern emerges in the Allais paradox where 35.3 percent of participants exhibit the intuitive paradoxical choice pattern by choosing A over B in I, but B over A in II (table 1ii). Conlisk's test rejects the hypothesis that violations of Independence are due to random, symmetric error $\left(\mathrm{Z}=6.92\right.$, $\mathrm{p}$-value $\left.<10^{-10}\right)$. Using similar proportions of non-zero prizes in a small-stakes Allais paradox experiment, Fan (2002) finds comparable results. Using different proportions of prizes, Huck and Müller (2012) administer a small-stake Allais paradox to a representative online panel of the Dutch population, and also find systematic violations, albeit less frequently. Table 5ii (appendix, p.25) shows that the results are robust across subsamples. Roughly speaking violations of Independence have similar prevalence as violations of Monotonicity do, in both cases about half the subjects violate the axioms, and in both cases the violations display the same asymmetric pattern. 
Violations of Monotonicity and Independence are related: The correlation between the two intuitive paradoxical choice patterns is 0.20 ( $\mathrm{p}$-value $<10^{-5}$ ). Table 2 presents a fuller picture of the relationship, giving the fraction of each of the possible 16 choice patterns across the four choice situations. With 18.3 percent, the most frequent choice pattern is to violate both Independence and Monotonicity in the intuitive way. The hypothesis, that behavior in the Monotonicity test is independent of behavior in the Allais paradox, is rejected (Pearson's chi-squared (9) $=151.24$, p-value $\left.<10^{-10}\right)$.

TABLE 2. Relationship between Independence and Monotonicity

\begin{tabular}{|c|c|c|c|c|c|}
\hline & & \multicolumn{4}{|c|}{ MONOTONICITY } \\
\hline & & \multicolumn{2}{|c|}{ Satisfy } & \multicolumn{2}{|c|}{ Violate } \\
\hline & & $\mathrm{AA}$ & $\mathrm{BB}$ & $\mathrm{AB}$ & $\mathrm{BA}$ \\
\hline \multirow{4}{*}{ 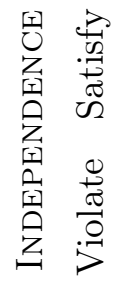 } & $\mathrm{AA}$ & 12.0 & 0.9 & 8.5 & 1.6 \\
\hline & $\mathrm{BB}$ & 5.6 & 9.6 & 7.8 & 3.4 \\
\hline & $\mathrm{AB}$ & 15.4 & 1.3 & 18.3 & 0.4 \\
\hline & $\mathrm{BA}$ & 3.8 & 3.4 & 4.2 & 3.8 \\
\hline
\end{tabular}

In this online study 28.4 percent of subjects violate AA-FOSD, which is substantially lower than the 48 percent of overall violations of Monotonicity. However, as AA-FOSD is a basic rationality axiom, a 28.4 percent violation rate is of concern. Although first-order stochastic dominance with respect to lotteries (FOSD) has been tested (e.g. von Winterfeldt, Chung, Luce, and Cho, 1997; Gneezy, List, and Wu, 2006; Charness, Karni, and Levin, 2007; Birnbaum, 2008; Sharma and Vadovic, 2015), we are unaware of any experimental tests of AA-FOSD. Satisfying AA-FOSD is more cognitively demanding than satisfying FOSD, as the Anscombe-Aumann framework of mixed objective/subjective uncertainty is more complex than a context of only objective uncertainty. It is thus difficult to know how to interpret these findings. Investigating this question is one of the motivations of study 2 .

4.2. Study 2 (Laboratory Experiment). Conducting a second study serves three purposes: First, study 1 finds that violations of AA-FOSD are common. As AA-FOSD is a basic rationality axiom this raises concerns about data quality. To address these concerns, study 2 takes place in the lab, and repeatedly administers the Monotonicity test to check for consistency of choices. Second, study 2 examines whether the results of study 1 robustly replicate with a different subject pool and different sources of uncertainty. Third, study 2 investigates three potential causes of the violations. 


\begin{tabular}{|llll|}
\hline CHF $\quad 0$ & Black (your color) & and & $1-9$ \\
\hline CHF 60 & Not black & and & $1-100$ \\
\hline CHF 100 & Black (your color) & and & $10-100$ \\
\hline
\end{tabular}

Figure 4. Visual representation of act 1B

Complexity aversion is a candidate, as the certain option in choice situation 1 in the Monotonicity test is less complex than its alternative. Another candidate explanation is that participants perceive Anscombe-Aumann acts as compound lotteries and fail to reduce them. Finally, violations could be due to the certainty effect. For this we are currently running another lab experiment.

4.2.1. Procedure. In the lab study we adapt the sources of uncertainty as follows: The source of objective uncertainty is a transparent envelope whose composition of 100 chips numbered from 1 to 100 is known to participants. Subjective uncertainty is implemented by an opaque envelope ("envelope A"). It contains 100 colored chips of unknown composition. Participants only know that each chip has exactly one of nine possible colors. At the beginning of the session each participant chooses one of the nine colors. At the end of the experiment, one chip from envelope A is drawn. If that chip has the color chosen by the participant, this corresponds to the realization of the event $E$ (horse 1-11 wins).

We replace the prizes of $\$ 1$ Million and $\$ 5$ Million by CHF 60, respectively CHF 100 (at the time of the experiment $1 \mathrm{CHF} \approx 1.03 \mathrm{USD})$. With these modifications, denoting $Q=\left(0 ; \frac{9}{100}, 100 ; \frac{91}{100}\right)$, and considering the event $E=$ "your chosen color" we get the experimental acts $1 \mathrm{~A}=\delta_{60}, 1 \mathrm{~B}=Q_{E} \delta_{60}$, $2 \mathrm{~A}=\delta_{60 E} \delta_{0}$, and $2 \mathrm{~B}=Q_{E} \delta_{0}$. Observation 1 applies. Figure 4 shows how acts are displayed to participants. This visual representation is carefully explained to the participants at the beginning of the experiment.

The Monotonicity test is administered twice. In addition we pose the following incentivized choices: the Allais paradox choices, and a choice on each FOSD and AA-FOSD. To measure complexity aversion, participants make two complexity aversion choices whose design follows Sonsino, Benzion, and Mador (2002) and Moffatt, Sitzia, and Zizzo (2015). To investigate whether participants fail to reduce compound lotteries, they are given a choice between a simple lottery and a compound lottery. The compound lottery is implemented by envelope $\mathrm{B}$, which is equivalent to 
the compound urn 3 Halevy (2007). To measure ambiguity aversion we give a two-color Ellsberg paradox, which employs envelope C, which is equivalent to the ambiguous urn 2 in Halevy (2007).

The sequence of the experiment is as follows: Participants of a session are assembled together and a subject is randomly selected. This subject does not make any choices, but blindfoldedly conducts all drawings at the end of the experiment. A set of the envelopes is shown and explained to the participants. Participants enter the lab where they find detailed printed instructions, the transparent, and the three opaque envelopes, A, B and C, on their desks. Participants are asked eight understanding questions, and get on-screen feedback. They then make the twelve incentivized choices, each on a separate screen. The RLI method is used to avoid hedging. On each screen the order of options is random. The first four choice screens display our Monotonicity test and the original Allais paradox in random order. Then the incentivized questions on FOSD, AA-FOSD, complexity aversion, failure to reduce compound lotteries and ambiguity aversion are posed in random order. The final two choices repeat the Monotonicity test, with the order of the choice options counterbalanced. This arrangement of the questions maximizes the distance between the first and second elicitations of Monotonicity in order to avoid memory effects. A demographic survey concludes the study. ${ }^{6}$

The experiment was run at the ETH DeSciL lab in May and June 2016. Subjects were students from the joint subject pool of the University of Zurich and the Swiss Federal Institute of Technology(ETH). The experiment was programmed in z-Tree (Fischbacher, 2007), participants were recruited with ORSEE (Greiner, 2015). The sessions lasted about forty minutes and average earnings were about $\$ 32$.

4.2.2. Results. Data from $N=72$ participants was collected. FOSD is violated by two subjects, and AA-FOSD is violated by two different subjects. So the rates of violations of FOSD and AA-FOSD are identical and very low (less than 3 percent). Note that the elicitation question of AA-FOSD in this study is designed to be as similar as possible to the one used in the online study. This lends support to the view that genuine violations of AA-FOSD are rare, and that violations in the online study are largely attributable to mistakes.

Table 3i provides participants' choices in the Monotonicity test. Choices in columns AA and $\mathrm{BB}$ do not violate Monotonicity, choices in columns $\mathrm{AB}$ and $\mathrm{BA}$ do. The first row of results corresponds to the full sample and the first elicitation. As in the online experiment the modal choice of participants violates Monotonicity: 41.7 percent of participants exhibit the intuitive paradoxical choice pattern AB. Violations of Monotonicity again exhibit an asymmetric pattern, and we can

\footnotetext{
${ }^{6}$ The web appendix contains the original instructions, translations, pictures of the lab setup and screenshots.
} 
TABLE 3. Violations of Monotonicity and Independence (in percent)

\begin{tabular}{|c|c|c|c|c|c|c|}
\hline & \multicolumn{2}{|c|}{ SATISFY } & \multicolumn{2}{|c|}{ VIOLATE } & \multirow[b]{2}{*}{ Conl.-Z } & \multirow[b]{2}{*}{$\mathrm{N}$} \\
\hline & AA & $\mathrm{BB}$ & $\mathrm{AB}$ & $\mathrm{BA}$ & & \\
\hline \multicolumn{7}{|l|}{ (i) Monotonicity } \\
\hline Full sample (first elicitation) & 23.6 & 29.2 & 41.7 & 5.6 & 5.20 & 72 \\
\hline Full sample (second elicitation) & 16.7 & 31.9 & 48.6 & 2.8 & 7.01 & 72 \\
\hline Consistent subjects & 13.9 & 33.3 & 52.8 & 0.0 & 6.25 & 36 \\
\hline Not complexity averse (first elic.) & 22.0 & 30.5 & 45.8 & 1.7 & 6.34 & 59 \\
\hline Satisfy ROCL (first elic.) & 16.7 & 36.7 & 40.0 & 6.7 & 3.01 & 30 \\
\hline Not ambiguity averse (first elic.) & 16.7 & 36.7 & 40.0 & 6.7 & 3.01 & 30 \\
\hline STEM major at ETH (first elic.) & 20.7 & 37.9 & 37.9 & 3.4 & 3.36 & 29 \\
\hline Satisfy FOSD and AA-FOSD (first elic.) & 25.0 & 27.9 & 41.2 & 5.9 & 4.91 & 68 \\
\hline \multicolumn{7}{|l|}{ (ii) Independence } \\
\hline Full sample & 6.9 & 45.8 & 43.1 & 4.2 & 5.78 & 72 \\
\hline
\end{tabular}

reject the null hypothesis that violations are due to symmetric, random error at all conventional significance levels (Conlisk's $\mathrm{Z}=5.2, \mathrm{p}$-value $<10^{-6}$ ). The second row gives the results for the full sample and the second elicitation. The paradox is more pronounced in the second elicitation than in the first one. The fraction of choices that are consistent across the elicitations, is 75 percent for choice situation 1 and 68 percent for choice situation 2, resulting in half of the subjects being consistent across elicitations for both choices. These rates of consistency are in line with the rates found in research of decision under risk (Stott, 2006, and survey table 1 therein; Baillon, Bleichrodt, and Cillo, 2015). Row three restricts the sample to those subjects who are consistent across elicitations: The intuitive paradoxical pattern is exhibited by 52.8 percent of these subjects, which is a higher fraction than for the full sample in either elicitation.

With the Conlisk-test the null hypothesis is that the violations are due to symmetric errors, i.e. error rates are the same in both choice situations. With repeated elicitation we can use the true-and-error model (Birnbaum et al. 2008, also see Regenwetter et al. 2010, Birnbaum et al., forthc.) which allows error rates to differ. With the true-and-error model, there are four possible types of true preferences in the experiment (AA, AB, BA, BB), and each type makes a mistake with probability $e_{1}$ in choice situation 1 , and with probability $e_{2}$ in choice situation $2 .^{7}$ The estimates for the error rates are $\hat{e_{1}}=0.15$ and $\hat{e_{2}}=0.20$. The shares of the types are estimated at 10 percent

\footnotetext{
${ }^{7}$ The true-and-error model assumes that the probability of a mistake does not vary by type or elicitation round. Moreover, it assumes that the errors are mutually independent across elicitation rounds and choice situations.
} 
for type AA, 61 percent for type AB, 0 percent for type BA, and 29 percent for type BB. Hence the true-and-error model raises the estimate of the probability that a subject's preferences feature the intuitive paradoxical choice pattern to 61 percent (95-percent confidence interval: $0.44-0.79$ ). Complete results are given in table 6 (appendix, p.26).

The remaining rows in table $3 \mathrm{i}$ repeat the analysis for subsamples. First, we consider the subsample of subjects who do not exhibit complexity aversion. The second subsample consists of those participants who do not fail to reduce compound lotteries. The remaining subsamples consider participants who do not exhibit ambiguity aversion, are students in science, technology, engineering or math (STEM) at ETH, or violate neither FOSD nor AA-FOSD. Results are remarkably similar across all subsamples. Hence, there is no evidence that violations can be explained by complexity aversion. Similarly, there is no evidence that violations are due to perception of the acts as compound lotteries combined with failure to reduce them. Interestingly, even among STEM majors, 37.9 percent display the intuitive paradoxical choice pattern.

The correlation between violations of reduction of compound lotteries and ambiguity aversion is of interest in itself, as Halevy (2007) suggests that the two are tightly associated. We find a correlation of 0.71, which is in line with the correlations Halevy (2007, robustness round) and Gillen, Snowberg, and Yariv (2015) report, but higher than what Abdellaoui, Klibanoff, and Placido (2015) find.

Table 3ii gives the results for the Allais paradox. The intuitive paradoxical choice pattern of A in choice I, B in choice II, is exhibited by 43.1 percent of participants, a fraction similar to the one found in the Monotonicity test, but the pattern is not modal. There is a positive correlation between these two intuitive paradoxical choice patterns of 0.18 . While not significant ( $\mathrm{p}$-value $=0.143$ ), it is remarkably similar to the one we find in study 1 where it is 0.20 . Table 4 supplies a detailed breakdown of the relationship: with 22.2 percent, the most frequent of the 16 possible choice patterns is to violate both Independence and Monotonicity in the intuitive way. However, we cannot reject the hypothesis that the behavior in the Monotonicity test is independent of that in the Allais paradox. These analyses use four choices per participant, so even small probabilities of mistakes substantially attenuate correlation measures.

One way to address this issue is to focus the analysis on those subjects for whom we have no evidence that they are making at least one mistake in an incentivized choice. If we take violations of FOSD, violations of AA-FOSD, and inconsistent choices to be mistakes, we are left with a subsample of $N=34$ participants who do not make a single mistake. In that subsample the intuitive paradoxical choice pattern is exhibited by 52.9 percent of participants for Monotonicity, and 44.1 percent for Independence. The correlation between the two intuitive paradoxical choice 
patterns is $0.36(\mathrm{p}$-value $=0.03)$. With 32.4 percent the most frequent choice pattern among the 16 possible ones, is to violate both Independence and Monotonicity in the intuitive way. The hypothesis that behaviors are independent is rejected (Pearson's chi-squared $(9)=15.8$, p-value $=0.07$ ).

TABLE 4. Relationship between Independence and Monotonicity

\begin{tabular}{|c|c|c|c|c|c|}
\hline & & \multicolumn{4}{|c|}{ MONOTONICITY } \\
\hline & & \multicolumn{2}{|c|}{ Satisfy } & \multicolumn{2}{|c|}{ Violate } \\
\hline & & $\mathrm{AA}$ & $\mathrm{BB}$ & $\mathrm{AB}$ & $\mathrm{BA}$ \\
\hline \multirow{4}{*}{ 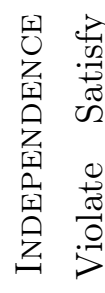 } & $\mathrm{AA}$ & 2.8 & 1.4 & 2.8 & 0.0 \\
\hline & $\mathrm{BB}$ & 11.1 & 16.7 & 16.7 & 1.4 \\
\hline & $\mathrm{AB}$ & 6.9 & 9.7 & 22.2 & 4.2 \\
\hline & $\mathrm{BA}$ & 2.8 & 1.4 & 0.0 & 0.0 \\
\hline
\end{tabular}

\section{Conclusion}

This paper studies Anscombe-Aumann's Monotonicity axiom by modifying the Allais paradox. While in the Allais paradox all uncertainty is objective, in our thought experiment and actual experiments some, but not all, uncertainty is subjective. The so-modified experiment ceases to be a test of Independence, and instead becomes a test of Monotonicity. In two studies we gather experimental evidence on the prevalence of violations of Monotonicity and compare it to the prevalence of violations of Independence.

Study 1 is conducted online, with a large number of subjects consisting of U.S. online workers and a natural source of ambiguity. Study 2 is run in the lab with European student subjects and the source of ambiguity is an Ellsberg urn. In both studies we find that the modal choice of subjects violates Monotonicity in a specific way, which we call the intuitive paradoxical choice pattern. The intuitive paradoxical choice pattern corresponds to the way Independence is typically violated in the Allais paradox. The prevalence of the intuitive paradoxical choice pattern is about 40 percent in both studies. Additionally, the Allais paradox is administered in both studies. The prevalence of the intuitive paradoxical choice pattern in the Allais paradox is also about 40 percent. Violations of Independence and Monotonicity are correlated.

A concern is that violations might be due to mistakes. In both studies, the hypothesis that violations are due to symmetric, random mistakes is easily rejected by the Conlisk-test. To further address this concern, study 2 poses the Monotonicity test twice. Among the subjects that choose 
consistently, the prevalence of the intuitive paradoxical choice pattern is 52.8 percent, which is higher than in the full sample. The repeated elicitation also allows us to estimate the parameters of a true-and-error model: the true prevalence of violations of Monotonicity is estimated at 61 percent.

Given frequent violations of Monotonicity, the question arises how they could be explained. The data suggest that neither complexity aversion nor perception of the Anscombe-Aumann acts as compound lotteries combined with failure to reduce them can account for the violations. To investigate whether the certainty effect can explain behavior, we are in the process of extending this paper by running another lab experiment.

As the descriptive validity of Monotonicity is comparable to that of Independence, Monotonicity is problematic as a universal descriptive assumption. There are several ways to respond to this problem. Viewing Monotonicity as descriptively problematic, Trautmann and Wakker (2015) propose an experimental approach which sidesteps Monotonicity. Our empirical evidence supports their view. In their approach, subjects are deliberately never confronted with mixed subjective/objective uncertainty. Their approach allows to test and apply models of ambiguity aversion that assume Monotonicity, even when it is known that subjects do not satisfy it. However, the limitation of their approach is that it cannot be used for evaluating predictions that rely on Monotonicity, such as invariability of ambiguity attitudes at low versus high outcomes (Machina, 2014), or concerning behavior in our thought experiment. There are several ways for models to capture richer behavior concerning such predictions. As Monotonicity is tailored to the AA-framework, models outside the AA-framework do not have problems with Monotonicity per se. But note that even outside the AA-framework, separability-related issues can arise, as Machina's (2009) thought experiments and an experimental implementation thereof by L'Haridon and Placido (2010) demonstrate. A notable exception is Segal's (1987) model: it can accommodate richer behavior in both our thought experiment and in the ones proposed in Machina (2009) and Machina (2014), see Dillenberger and Segal, 2015. However, to capture modal behavior in our experiment, leaving the AA-framework is not necessary: Models within the AA-framework can drop or relax Monotonicity. A candidate axiom, to replace Monotonicity, is first-order stochastic dominance generalized to the AA-framework (AAFOSD). This is the approach taken by Bommier (2016). To our knowledge, our studies are the first to test AA-FOSD rather than merely FOSD. Both studies suggest that AA-FOSD is descriptively more plausible than Monotonicity. In the lab experiment, violations of AA-FOSD are rare. This provides support for descriptive models that assume AA-FOSD. 


\section{REFERENCES}

Abdellaoui, M., P. Klibanoff, and L. Placido (2015): "Experiments on Compound Risk in Relation to Simple Risk and to Ambiguity," Management Science, 61(6), 1306-1322.

Allais, M. (1953): "Le Comportement de l'Homme Rationnel devant le Risque: Critique des Postulats et Axiomes de l'Ecole Americaine," Econometrica, 21(4), pp. 503-546.

Allais, M. (1979): The Foundations of a Positive Theory of Choice Involving Risk and a Criticism of the Postulates and Axioms of the American School (1952)pp. 27-145. Springer Netherlands, Dordrecht.

Anscombe, F. J., and R. J. Aumann (1963): "A Definition of Subjective Probability," Annals of Mathematical Statistics, 34(1), pp. 199-205.

Baillon, A., H. Bleichrodt, And A. Cillo (2015): "A Tailor-Made Test of Intransitive Choice," Operations Research, 63(1), 198-211.

Birnbaum, M. H. (2008): "New paradoxes of risky decision making," Psychological Review, 115(2), $463-501$.

Birnbaum, M. H., D. Navarro-Martinez, C. Ungemach, N. Stewart, and E. G. QuispeTorreblanca (2016): "Risky decision making: Testing for violations of transitivity predicted by an editing mechanism," Judgment and Decision Making, 11(1), 75-91.

Birnbaum, M. H., And U. Schmidt (2008): "An experimental investigation of violations of transitivity in choice under uncertainty," Journal of Risk and Uncertainty, 37(1), 77-91.

Birnbaum, M. H., U. Schmidt, and M. D. Schneider (forthcoming): "Testing independence conditions in the presence of errors and splitting effects," Journal of Risk and Uncertainty.

Bleichrodt, H., And P. P. Wakker (2015): "Regret Theory: A Bold Alternative to the Alternatives," Economic Journal, 125(583), 493-532.

Bommier, A. (2016): "A Dual Approach to Ambiguity Aversion," Discussion paper, CER-ETH Center of Economic Research at ETH Zurich.

Cerreia-Vioglio, S., P. Ghirardato, F. Maccheroni, M. Marinacci, and M. Siniscalchi (2011): "Rational preferences under ambiguity," Economic Theory, 48(2-3), 341-375.

Cerreia-Vioglio, S., F. Maccheroni, M. Marinacci, and L. Montrucchio (2011): "Uncertainty averse preferences," Journal of Economic Theory, 146(4), 1275-1330.

Charness, G., E. Karni, And D. Levin (2007): "Individual and group decision making under risk: An experimental study of Bayesian updating and violations of first-order stochastic dominance," Journal of Risk and Uncertainty, 35(2), 129-148. 
Chateauneuf, A., and J. H. Faro (2009): "Ambiguity through confidence functions," Journal of Mathematical Economics, 45(9 - 10), 535-558.

Conlisk, J. (1989): "Three Variants on the Allais Example," American Economic Review, 79(3), pp. 392-407.

Dean, M., And P. Ortoleva (forthcoming): "Allais, Ellsberg, and Preferences for Hedging," Theoretical Economics.

Dillenberger, D., And U. Segal (2015): "Recursive Ambiguity and Machina's examples," International Economic Review, 56(1), 55-61.

EllsberG, D. (1961): "Risk, Ambiguity, and the Savage Axioms," Quarterly Journal of Economics, 75(4), pp. 643-669.

FAN, C. (2002): "Allais paradox in the small," Journal of Economic Behavior and Organization, 49(3), 411-421.

FischBACHER, U. (2007): "z-Tree: Zurich toolbox for ready-made economic experiments," Experimental Economics, 10(2), 171-178.

Fox, C. R., And A. Tversky (1995): "Ambiguity Aversion and Comparative Ignorance," Quarterly Journal of Economics, 110(3), 585-603.

Gilboa, I., And M. Marinacci (2013): "Ambiguity and the Bayesian Paradigm," in Advances in Economics and Econometrics: Tenth World Congress, ed. by E. Society, E. S. W. Congress, D. Acemoglu, M. Arellano, and E. Dekel, Advances in Economics and Econometrics 3 Volume Hardback Set, chap. 7, pp. 179-242. Cambridge University Press.

GilboA, I., And D. Schmeidler (1989): "Maxmin expected utility with non-unique prior," Journal of Mathematical Economics, 18(2), 141-153.

Gillen, B., E. Snowberg, and L. Yariv (2015): "Experimenting with Measurement Error: Techniques with Applications to the Caltech Cohort Study," Working Paper 21517, National Bureau of Economic Research.

Gneezy, U., J. A. List, And G. Wu (2006): "The Uncertainty Effect: When a Risky Prospect is Valued Less than its Worst Possible Outcome," Quarterly Journal of Economics, 121(4), 12831309.

Grant, S., AND B. POlAK (2013): "Mean-dispersion preferences and constant absolute uncertainty aversion," Journal of Economic Theory, 148(4), 1361-1398.

Greiner, B. (2015): "Subject pool recruitment procedures: organizing experiments with ORSEE," Journal of the Economic Science Association, 1(1), 114-125.

Halevy, Y. (2007): "Ellsberg Revisited: An Experimental Study," Econometrica, 75(2), 503-536. 
Hey, J. D. (2014): "Chapter 14 - Choice under Uncertainty: Empirical Methods and Experimental Results," in Handbook of the Economics of Risk and Uncertainty, ed. by M. Machina, and K. Viscusi, vol. 1, pp. 809-850. North-Holland.

HuCK, S., AND W. MÜLLER (2012): "Allais for all: Revisiting the paradox in a large representative sample," Journal of Risk and Uncertainty, 44(3), 261-293.

Kahneman, D., And A. Tversky (1979): "Prospect Theory: An Analysis of Decision under Risk," Econometrica, 47(2), pp. 263-292.

Klibanoff, P., M. Marinacci, and S. Mukerji (2005): "A Smooth Model of Decision Making under Ambiguity," Econometrica, 73(6), pp. 1849-1892.

L'Haridon, O., and L. Placido (2010): "Betting on Machinas reflection example: an experiment on ambiguity," Theory and Decision, 69(3), 375-393.

Loomes, G., And R. Sugden (1982): "Regret Theory: An Alternative Theory of Rational Choice Under Uncertainty," The Economic Journal, 92(368), 805-824.

Maccheroni, F., M. Marinacci, and A. Rustichini (2006): "Ambiguity Aversion, Robustness, and the Variational Representation of Preferences," Econometrica, 74(6), pp. 1447-1498.

MacCrimmon, K., and S. Larsson (1979): "Utility Theory: Axioms Versus Paradoxes," in Expected Utility Hypotheses and the Allais Paradox, ed. by M. Allais, and O. Hagen, vol. 21 of Theory and Decision Library, pp. 333-409. Springer Netherlands.

Machina, M. J. (1989): "Dynamic Consistency and Non-Expected Utility Models of Choice Under Uncertainty," Journal of Economic Literature, 27(4), pp. 1622-1668.

- (2009): "Risk, Ambiguity, and the Rank-Dependence Axioms," The American Economic Review, 99(1), pp. 385-392.

- (2014): "Ambiguity Aversion with Three or More Outcomes," American Economic Review, 104(12), 3814-40.

Machina, M. J., And D. Schmeidler (1995): "Bayes without Bernoulli: Simple Conditions for Probabilistically Sophisticated Choice," Journal of Economic Theory, 67(1), 106-128.

Moffatt, P. G., S. Sitzia, And D. J. Zizzo (2015): "Heterogeneity in preferences towards complexity," Journal of Risk and Uncertainty, 51(2), 147-170.

Quigain, J. (1982): "A theory of anticipated utility," Journal of Economic Behavior E Organization, 3(4), 323-343.

Regenwetter, M., J. Dana, and C. P. Davis-Stober (2010): "Testing Transitivity of Preferences on Two-Alternative Forced Choice Data," Frontiers in Psychology, 1, 1-15. 
Schmeidler, D. (1989): "Subjective Probability and Expected Utility without Additivity," Econometrica, 57(3), pp. 571-587.

SEgAL, U. (1987): "The Ellsberg paradox and risk aversion: An anticipated utility approach," International Economic Review, 28(1), 175-202.

Sharma, T., and R. Vadovic (2015): "Dominated Choices," Discussion paper.

Siniscalchi, M. (2009): "Vector Expected Utility and Attitudes Toward Variation," Econometrica, $77(3), 801-855$.

SkIADAS, C. (2013): "Scale-invariant uncertainty-averse preferences and source-dependent constant relative risk aversion," Theoretical Economics, 8(1), 59-93.

Slovic, P., And A. Tversky (1974): "Who accepts Savage's axiom?," Behavioral Science, 19(6), $368-373$.

Sonsino, D., U. Benzion, and G. Mador (2002): "The Complexity Effects on Choice with Uncertainty Experimental Evidence," The Economic Journal, 112(482), 936-965.

Sтотт, H. P. (2006): "Cumulative prospect theory's functional menagerie," Journal of Risk and Uncertainty, 32(2), 101-130.

Trautmann, S., And P. P. Wakker (2015): "Making the Anscombe-Aumann Approach to Ambiguity Suited for Descriptive Applications," Discussion paper, Erasmus University Rotterdam.

Trautmann, S. T., And G. van De Kuilen (2015): "Ambiguity Attitudes," in Handbook of Judgment and Decision Making, ed. by G. Keren, and G. Wu. Blackwell.

Tversky, A., And D. Kahneman (1992): "Advances in prospect theory: Cumulative representation of uncertainty," Journal of Risk and Uncertainty, 5(4), 297-323.

von Winterfeldt, D., N.-K. Chung, D. R. Luce, and Y. Cho (1997): "Tests of Consequence Monotonicity in Decision Making Under Uncertainty," Journal of Experimental Psychology Learning Memory and Cognition, 23(2), 406-426.

Wakker, P. P. (2010): Prospect Theory: For Risk and Ambiguity. Cambridge University Press. (2011): "Jaffray's ideas on ambiguity," Theory and Decision, 71(1), 11-22.

Wu, G., and R. Gonzalez (1999): "Nonlinear Decision Weights in Choice Under Uncertainty," Management Science, 45(1), 74-85.

\section{Appendix A: Proofs}

Proof of observation 1: Observation 1 is equivalent to the following statement:

If $\succsim$ satisfies Weak order and Monotonicity, then (i) $1 \mathrm{~A} \succ 1 \mathrm{~B}$ implies $2 \mathrm{~A} \succsim 2 \mathrm{~B}$, (ii) $1 \mathrm{~B} \succ 1 \mathrm{~A}$ implies $2 \mathrm{~B} \succsim 2 \mathrm{~A}$, (iii) $2 \mathrm{~A} \succ 2 \mathrm{~B}$ implies $1 \mathrm{~A} \succsim 1 \mathrm{~B}$, and (iv) $2 \mathrm{~B} \succ 2 \mathrm{~A}$ implies $1 \mathrm{~B} \succsim 1 \mathrm{~A}$. 
Proof of (i): First, observe that $1 \mathrm{~A} \succ 1 \mathrm{~B}$ implies $\delta_{1} \succ P$, as otherwise we would have $P \succsim \delta_{1}$ which by Monotonicity implies 1B $\succsim 1 \mathrm{~A}$, a contradiction. Second, by Monotonicity, $\delta_{1} \succ P$ implies $2 \mathrm{~A} \succsim 2 \mathrm{~B}$.

The proofs of (ii) - (iv) are analogous.

Equivalence of AA-Independence and Independence under probabilistic sophistication:

Axiom (AA-Independence over $M)$. For all acts $f, g, h$ in $\mathcal{F}$, and for all $\alpha$ in $M \subseteq(0,1)$ :

$$
f \succsim g \Rightarrow \alpha f+(1-\alpha) h \succsim \alpha g+(1-\alpha) h .
$$

Lemma 1. If $\succsim$ satisfies Weak order and Probabilistic sophistication then for all $M \subseteq(0,1)$ : Independence over $M$ is equivalent to AA-Independence over $M$.

Proof: AA-Independence over $M$ trivially implies Independence over $M$.

Under probabilistic sophistication Independence over $M$ implies Independence over $M$ :

Consider $f, g, h$ in $\mathcal{F}$, and $\alpha$ in $M$, with $f \succsim g$. Take the $\mu$ arising from Probabilistic sophistication, and denote the partitions $\mathcal{E}(f)=\left(F_{1}, \ldots, F_{I}\right), \mathcal{E}(g)=\left(G_{1}, \ldots, G_{J}\right), \mathcal{E}(h)=\left(H_{1}, \ldots, H_{K}\right), \mathcal{E}(\alpha f+$ $(1-\alpha) h)=\left(F^{\prime}{ }_{1}, \ldots, F_{L}^{\prime}\right)$ and $\mathcal{E}(\alpha g+(1-\alpha) h)=\left(G^{\prime}{ }_{1}, \ldots, G_{M}^{\prime}\right)$, then define $P=\sum_{i=1}^{I} \mu\left(F_{i}\right) f\left(F_{i}\right)$, $Q=\sum_{j=1}^{J} \mu\left(G_{j}\right) g\left(G_{j}\right), R=\sum_{k=1}^{K} \mu\left(H_{k}\right) h\left(H_{k}\right), P^{\prime}=\sum_{l=1}^{L} \mu\left(F_{l}^{\prime}\right)(\alpha f+(1-\alpha) h)\left(F_{l}^{\prime}\right)$ and $Q^{\prime}=$ $\sum_{m=1}^{M} \mu\left(G_{m}^{\prime}\right)(\alpha g+(1-\alpha) h)\left(G_{m}^{\prime}\right)$. Note that these acts are constructed such that under Probabilistic sophistication $f \sim P, g \sim Q, \alpha f+(1-\alpha) h \sim P^{\prime}$ and $\alpha g+(1-\alpha) h \sim Q^{\prime}$. Independence over $M$ implies $\alpha P+(1-\alpha) R \succsim \alpha Q+(1-\alpha) R$. By countable additivity of $\mu$ we have $\alpha P+(1-\alpha) R=P^{\prime}$ and $\alpha Q+(1-\alpha) R=Q^{\prime}$.

\section{Proof of proposition 1:}

By Lemma 1 it suffices to show that AA-Independence over $M_{\mu}$ is equivalent to Monotonicity:

AA-Independence over $M_{\mu}$ implies Monotonicity:

Consider two constant acts $P, Q$ with $P \succsim Q$. It suffices to show that for any act $h$ and any event $E \in \Sigma: P_{E} h \succsim Q_{E} h$.

Take the $\mu$ arising from Probabilistic sophistication, and the partition $\mathcal{E}\left(P_{E} h\right)=\mathcal{E}\left(Q_{E} h\right)=$ $\left(E, E_{1}^{\prime}, \ldots E_{J}^{\prime}\right)$, then $P_{E} h \sim \mu(E) P+(1-\mu(E)) R$, where $R=\sum_{j=1}^{J} \frac{\mu\left(E_{j}^{\prime}\right)}{1-\mu(E)} h_{j}$. By an analogous argument we have $Q_{E} h \sim \mu(E) Q+(1-\mu(E)) R$, where $R$ is defined as before. Now note that $\mu(E) \in M_{\mu}$, therefore by AA-Independence over $M_{\mu}$ :

$$
\mu(E) P+(1-\mu(E)) R \succsim \mu(E) Q+(1-\mu(E)) R .
$$

Monotonicity implies AA-Independence over $M_{\mu}$ : 
Consider three acts $f, g, h$ with $f \succsim g$ and any $\alpha \in M_{\mu}$. We have to show that $\alpha f+(1-\alpha) h \succsim$ $\alpha g+(1-\alpha) h$.

Consider the $\mu$ arising from Probabilistic sophistication. As $\alpha \in M_{\mu}$, there exists an event $A \in \Sigma$ s.t. $\mu(A)=\alpha$.

Denote $\mathcal{E}(\alpha f+(1-\alpha) h)=\left(E_{1}, \ldots, E_{N}\right)$ and define $P=\sum_{i=1}^{N} \mu\left(E_{i}\right) f\left(E_{i}\right)$, and $R=\sum_{i=1}^{N} \mu\left(E_{i}\right) h\left(E_{i}\right)$. Therefore by definition of $\mu$, we have $\alpha f+(1-\alpha) h \sim \sum_{i=1}^{N} \mu\left(E_{i}\right)\left[\alpha f\left(E_{i}\right)+(1-\alpha) h\left(E_{i}\right)\right]=$ $\alpha P+(1-\alpha) R$. By applying probabilistic sophistication we obtain $P_{A} R \sim \alpha P+(1-\alpha) R$, thus $P_{A} R \sim \alpha f+(1-\alpha) h$.

Denote $\mathcal{E}(\alpha g+(1-\alpha) h)=\left(E_{1}^{\prime}, \ldots, E_{J}^{\prime}\right)$ and define $Q=\sum_{j=1}^{J} \mu\left(E_{j}^{\prime}\right) g\left(E_{j}^{\prime}\right)$, and $R^{\prime}=\sum_{j=1}^{J} \mu\left(E_{j}^{\prime}\right) h\left(E_{j}^{\prime}\right)$.

Proceeding as before we get $Q_{A} R^{\prime} \sim \alpha g+(1-\alpha) h$.

By countable additivity of $\mu$ we have $R=R^{\prime}$, therefore $Q_{A} R^{\prime}=Q_{A} R$. It remains to be shown that $P_{A} R \succsim Q_{A} R$, which is implied by Monotonicity as $P \succsim Q$. 


\section{Appendix B1: Study 1 - Further experimental Results}

TABLE 5. Violations of Monotonicity and Independence: Robustness Checks (in percent)

\begin{tabular}{|c|c|c|c|c|c|c|}
\hline & \multicolumn{2}{|c|}{ SATisfy } & \multicolumn{2}{|c|}{ Violate } & \multirow[b]{2}{*}{ Conl.-Z } & \multirow[b]{2}{*}{$\mathrm{N}$} \\
\hline & AA & $\mathrm{BB}$ & $\mathrm{AB}$ & BA & & \\
\hline \multicolumn{7}{|l|}{ (i) Monotonicity } \\
\hline Full Sample & 36.8 & 15.2 & 38.8 & 9.2 & 11.06 & 552 \\
\hline Testq. 1 correct & 37.1 & 14.9 & 38.9 & 9.1 & 10.25 & 463 \\
\hline Testq. 2 correct & 37.1 & 14.8 & 39.3 & 8.8 & 10.97 & 501 \\
\hline Testq. 3 correct & 38.4 & 14.5 & 38.7 & 8.5 & 8.71 & 318 \\
\hline Testq. $1-2$ correct & 36.7 & 14.7 & 39.7 & 8.9 & 10.24 & 436 \\
\hline Testq. 1-3 correct & 37.6 & 14.5 & 40.5 & 7.4 & 8.44 & 242 \\
\hline Testq. 1-2 correct \& AA-FOSD & 35.3 & 17.2 & 38.1 & 9.4 & 8.33 & 331 \\
\hline Testq. 1-3 correct \& AA-FOSD & 35.6 & 17.5 & 37.9 & 9.0 & 6.15 & 177 \\
\hline 4. q.; Testq. 1-2 cor. \& AA-FOSD & 34.0 & 20.2 & 37.2 & 8.5 & 4.52 & 94 \\
\hline 4. q.; Testq. 1-3 cor. \& AA-FOSD & 35.3 & 21.6 & 37.3 & 5.9 & 3.84 & 51 \\
\hline Belief $\leq 10 \%$ & 35.8 & 16.9 & 39.8 & 7.5 & 8.46 & 254 \\
\hline Belief $>10 \%$ & 37.2 & 14.3 & 37.2 & 11.3 & 6.10 & 231 \\
\hline \multicolumn{7}{|l|}{ (ii) Independence } \\
\hline Full Sample & 23.0 & 26.4 & 35.3 & 15.2 & 6.92 & 552 \\
\hline Testq. 1 correct & 23.8 & 25.3 & 35.4 & 15.6 & 6.23 & 463 \\
\hline Testq. 2 correct & 24.4 & 25.3 & 35.3 & 15.0 & 6.70 & 501 \\
\hline Testq. 3 correct & 25.2 & 21.7 & 37.4 & 15.7 & 5.55 & 318 \\
\hline Testq. 1-2 correct & 24.5 & 24.8 & 35.3 & 15.4 & 6.09 & 436 \\
\hline Testq. $1-3$ correct & 28.9 & 17.8 & 36.8 & 16.5 & 4.48 & 242 \\
\hline Satisfy AA-FOSD & 23.0 & 26.1 & 33.9 & 17.0 & 4.86 & 395 \\
\hline Testq. 1-2 correct \& AA-FOSD & 23.9 & 25.7 & 32.9 & 17.5 & 4.04 & 331 \\
\hline Testq. 1-3 correct \& AA-FOSD & 28.8 & 17.5 & 33.9 & 19.8 & 2.61 & 177 \\
\hline Duration 1. quartile & 21.7 & 28.3 & 37.7 & 12.3 & 4.50 & 138 \\
\hline Duration 2. quartile & 21.7 & 21.7 & 39.1 & 17.4 & 3.54 & 138 \\
\hline Duration 3. quartile & 21.7 & 29.0 & 34.1 & 15.2 & 3.26 & 138 \\
\hline Duration 4. quartile & 26.8 & 26.8 & 30.4 & 15.9 & 2.55 & 138 \\
\hline 4. q.; Testq. 1-2 cor. \& AA-FOSD & 25.5 & 29.8 & 26.6 & 18.1 & 1.24 & 94 \\
\hline 4. q.; Testq. $1-3$ cor. \& AA-FOSD & 29.4 & 23.5 & 29.4 & 17.6 & 1.23 & 51 \\
\hline
\end{tabular}


APPENDIX B2: STUdy 2 - FURTHER EXPERIMENTAL RESUlTS

TABLE 6. True-and-error Model

\begin{tabular}{|c|c|c|}
\hline Parameter & Estimate & $\mathrm{CI}$ \\
\hline$p_{A A}$ & 10 & $0-22$ \\
\hline$p_{A B}$ & 61 & $44-79$ \\
\hline$p_{B A}$ & 0 & $0-0$ \\
\hline$p_{B B}$ & 29 & $16-42$ \\
\hline$e_{1}$ & 15 & $10-24$ \\
\hline$e_{2}$ & 20 & $14-30$ \\
\hline$\chi^{2}$ & 8.31 & \\
\hline
\end{tabular}

Birnbaum et al. (2016) 